Pacific Journal of Mathematics

SOME THEOREMS IN FOURIER ANALYSIS ON SYMMETRIC
SETS 


\section{SOME THEOREMS IN FOURIER ANALYSIS ON SYMMETRIC SETS}

\section{R. SCHNEIDER}

Let $R$ be the real line and $A=A(R)$ the space of continuous functions on $R$ which are the Fourier transforms of functions in $L^{1}(R) . \quad A(R)$ is a Banach Algebra when it is given the $L^{1}(R)$ norm. For a closed $F \cong R$ one defines $A(F)$ as the restrictions of $f \in A$ to $F$ with the norm of $g \in A(F)$ the infimum of the norms of elements of $A$ whose restrictions are $g$. Let $F_{r} \leqq R$ be of the form

$$
F_{r}=\left\{\sum_{1}^{\infty} \varepsilon_{j} r(j): \varepsilon_{j} \text { either } 0 \text { or } 1\right\} .
$$

This paper shows that if

$$
\sum(r(j+1) / r(j))^{2}<\infty \text { and } \sum(s(j+1) / s(j))^{2}<\infty
$$

then $A\left(F_{r}\right)$ is isomorphic to $A\left(F_{s}\right)$. We also show that, in some sense square summability is the best possible criterion. In the course of the proof we show that $F_{r}$ is a set of synthesis and uniqueness if $\sum(r(j+1) / r(j))^{2}<\infty$. This is almost a converse to a theorem of Salem.

We shall also consider sets $E_{m} \cong \Pi_{1}^{\infty} Z_{m(j)}$ of the form

$$
E_{m}=\left\{x: j^{\text {th }} \text { coordinate is } 0 \text { or } 1\right\} \text {. }
$$

The $E_{m}$ will have analogous properties to the $F_{r}$ that will depend on the $m(j)$.

The original work on isomorphisms of the algebras was done in [2] where Beurling and Helson show that any automorphism of $A$ must arise from a map $\varphi$ by $f \circ \varphi$ where $\varphi(x)=a x+b$. For restriction algebra the situation is more complex. In [5] it is shown that an isomorphism between $A\left(F_{1}\right)$ and $A\left(F_{2}\right)$ of norm one must be given by $f \rightarrow f \circ \varphi$ where $\varphi: F_{2} \rightarrow F_{1}$ is continuous and $e^{i \varphi}$ is a restriction to $F_{2}$ of a character of the discrete reals. Further if $F_{2}$ is thick in some appropriate sense the character is continuous. However, McGehee [11] gives examples of $F_{1}$ and $F_{2}$ for which the restriction algebras $A\left(F_{1}\right)$ and $A\left(F_{2}\right)$ are isomorphic under an isomorphism induced by a discontinuous character. Meyer [12] has shown that if

$$
\sum r(j+1) / r(j)<\infty \text { and } \sum s(j+1) / s(j)<\infty
$$

then $A\left(F_{r}\right)$ is isomorphic to $A\left(F_{s}\right)$. For appropriate $r(j)$ this is an example of an isomorphism induced by a $\varphi$ with $e^{i \varphi}$ not even a discontinuous character. He also showed that under these hypothesis $F_{r}$ was a set of synthesis and uniqueness. 
Definitions AND Notations. For background material and notation not defined here we refer the reader to [7] and [15].

In this paper $G$ will always be a locally compact abelian group with dual group $\Gamma$. If $g$ and $\gamma$ are elements of $G$ and $\Gamma$ respectively, the value of the character $\gamma$ at the point $g$ will be denoted by $(\gamma, g)$.

When we have a sequence of compact abelian groups $G_{j}$, we shall denote their direct product (complete direct sum [15]) by $\Pi G_{j}$. If $\Gamma_{j}$ is the dual of $G_{j}$, then the direct sum [15] $\Sigma \Gamma_{j}$ is the dual of $\Pi G_{j}$. The $j^{t h}$ coordinate of elements $g$ of $\Pi G_{j}$ or $\gamma$ of $\Sigma \Gamma_{j}$ will be denoted by $g_{j}$ and $\gamma_{j}$. One has:

$$
(\gamma, g)=\Pi\left(\gamma_{j}, g_{j}\right)
$$

where all but a finite number of elements in the product are 1 .

We shall be dealing with the following basic groups:

(i) The multiplicative circle group will be denoted by $T . T$ shall be identified with the unit interval by $x \in[0,1) \rightarrow \exp (x)$ where $\exp (x)=e^{2 \pi i x}$. The additive group of integers $Z$ is the dual group of $T$. If $x \in[0,1)$ represents an element of $T$ and $n \in Z$ then $(n, x)=$ $\exp (n x)$.

(ii) $R$ will denote the additive group of reals. $R$ is isomorphic to its dual under the pairing given by

$$
(y, x)=\exp (x y)
$$

$x, y \in R$.

(iii) $Z_{n}$ for $n \geqq 2$ will denote the additive group of integers $\bmod n . Z_{n}$ is also isomorphic to its dual under the pairing given by

$$
(r, s)=\exp (r s / n)
$$

$\mathrm{r}, s \in Z_{n}$.

Any nonzero regular translation invariant measure on a locally compact abelian group $G$ is called a Haar measure. If $\mu_{G}$ and $\mu_{T}$ are Haar measures on $G$ and its dual group $\Gamma$ respectively, the Fourier transform $\hat{f}$ of $f$ in $L^{1}\left(\Gamma, \mu_{\Gamma}\right)$ is defined by

$$
\hat{f}(g)=\int_{\Gamma} f(\gamma)(\gamma, g) d \mu_{\Gamma}
$$

for $g \in G$. The inversion theorem gives

$$
\int_{G} \hat{f}(g)(\gamma,-g) d \mu_{G}=C f(\gamma) .
$$

We shall normalize $\mu_{G}$ and $\mu_{\Gamma}$ so that $C=1$. If $G$ is compact we can place $\mu_{G}(G)=1$ and if $\Gamma$ is discrete $\mu_{\Gamma}(\gamma)=1$ for $\gamma \in \Gamma . L^{1}(G)$ will denote $L^{1}\left(G, \mu_{G}\right)$ for a normalized Haar measure. 
For $f, h \in L^{1}(\Gamma)$ define the convolution $f_{*} h$ by

$$
f * h(\gamma)=\int_{\lambda \in \Gamma} f(\gamma-\lambda) h(\lambda) d \mu_{\Gamma} .
$$

In [15] it is shown that $L^{1}(\Gamma)$ is a commutative Banach algebra under convolution and for $g \in G$

$$
\widehat{f * h(g)}=\hat{f}(g) \hat{h}(g) \text {. }
$$

We denote by $M(G)$ the space of all regular, complex valued Borel measures on $G$ of finite total variation. In [15] the Fourier transform $\hat{\mu}$ of $\mu \in M(G)$ and the convolution $\mu * \nu$ of measures in $M(G)$ are defined. It is shown that $M(G)$ is a Commutative Banach Algebra under convolution and

$$
\widehat{\mu * \nu}(\gamma)=\hat{\mu}(\gamma) \cdot \hat{\nu}(\gamma)
$$

for $\gamma \in \Gamma$.

Let $A=A(G)$ be defined by

$$
A(G)=\left\{\hat{f}: f \in L^{1}(\Gamma)\right\} .
$$

$A(G)$ is a Banach algebra under pointwise multiplication and with norm $\|\cdot\|_{A}$ defined by $\|\hat{f}\|_{A}=\|f\|_{L^{1}(\Gamma)}$ and is isomorphic to $L^{1}(\Gamma)$ under*. For a closed set $E \subseteq G$ define the restriction algebra

$$
A(E)=\left\{\hat{f} / E: f \in L^{1}(\Gamma)\right\}
$$

with norm $\|\cdot\|_{A(E)}$ defined by

$$
\|h\|_{A(E)}=\inf \left\{\|\hat{f}\|_{A}: \hat{f} / E=h\right\} \text {. }
$$

$A(E)$ is again a Banach algebra under pointwise multiplication. Set

$$
I(E)=\left\{\hat{f}: \hat{f} / E=0 \quad \text { and } \quad f \in L^{1}(\Gamma)\right\}
$$

$A(E)$ can be identified with the quotient algebra $A(G) / I(E)$.

The dual space of $A(G)$ is denoted by $P M$ (or $P M(G)$ ). Its elements are called pseudomeasures. Each $S \in P M$ can be identified with a function $\hat{S} \in L^{\infty}(\Gamma)$ as follows. The action of $S \in P M$ as a linear functional on $\hat{f} \in A(G)$ is given by

$$
(S, \hat{f})=\int_{r} f(\gamma) \overline{\hat{S}(\gamma)} d \mu_{\Gamma}
$$

We shall denote by $\|S\|_{P M}$ the $L^{\infty}(\Gamma)$ norm of $\hat{S}$. Thus $P M$ under $\|\cdot\|_{P M}$ is identical with $L^{\infty}(\Gamma)$ under the sup norm.

Since $A(E)$ is the quotient of $A(G)$ by $I(E)$, the dual of $A(E)$ consists of those $S \in P M$ which annihilate every function in $I(E)$. 
We shall denote this dual of $A(E)$ by $N(E)$. If $N(E)$ is the set of all $S \in P M$ with supp $S \subseteq E[7$, p.161], then $E$ is said to be a set of synthesis. The set of all $\mu \in M(G)$ with support in $E$ we denote by $M(E)$. $M(E)$ can be considered a subspace of $N(E)$ with $(\mu, \hat{f})=$ $\int \hat{f} d \bar{\mu}$. The two definitions for $\hat{\mu}$ coincide.

If $G_{1}$ and $G_{2}$ are locally compact abelian groups and $E_{1}$ and $E_{2}$ are closed subsets of $G_{1}$ and $G_{2}$ respectively we say that $\Phi: A\left(E_{1}\right) \rightarrow A\left(E_{2}\right)$ is an isomorphism into if and only if it is an injective algebraic homomorphism and is continuous. If the range of $\Phi$ is dense in $A\left(E_{2}\right)$ there exists a continuous $\varphi: E_{2} \rightarrow E_{1}$ with $\Phi f=f \circ \varphi$ [9]. We always denote the adjoint of $\Phi$ taking $N\left(E_{2}\right)$ into $N\left(E_{1}\right)$ by $\Phi^{*}$.

Symmetric sets in $R$ are defined as follows. For any sequence $r=\{r(j): j=1, \cdots\}$ of positive reals with the property

$$
\sum_{k}^{\infty} r(j)<r(k-1)
$$

we define the subset $F_{r}$ of $R$ by

$$
F_{r}=\left\{\sum_{1}^{\infty} \varepsilon_{j} r(j): \varepsilon_{j} \text { either } 0 \text { or } 1\right\} .
$$

The representation of the elements of $F_{r}$ as an infinite sum is unique. For each positive integer $k$, the subset $F_{r}^{k}$ or $F_{r}$ is defined by

$$
F_{r}^{k}=\left\{\sum_{1}^{k} \varepsilon_{j} r(j): \varepsilon_{j} \text { either } 0 \text { or } 1\right\} \text {. }
$$

We define the subspace $N_{1}\left(F_{r}\right)$ of $N\left(F_{r}\right)$ by

$$
N_{1}\left(F_{r}\right)=\bigcup_{k=1}^{\infty} M\left(F_{r}^{k}\right) \text {. }
$$

For any given sequence $m=\{m(j): j=1,2, \cdots\}$ of positive integers we define the subset $E_{m}$ of $\Pi_{j} Z_{m(j)}$ by

$$
E_{m}=\left\{x: x \in \Pi Z_{m(j)} ; x_{j} \text { either } 0 \text { or } 1\right\} \text {. }
$$

For each positive integer $k$ the subset $E_{m}^{k}$ of $E_{m}$ is defined by

$$
E_{m}^{k}=\left\{x: x \in E_{m} ; x_{j}=0 \text { if } j>k\right\} \text {. }
$$

Define the subspace $N_{1}\left(E_{m}\right)$ of $N\left(E_{m}\right)$ by

$$
N_{1}\left(E_{m}\right)=\bigcup_{k=1}^{\infty} M\left(E_{m}^{k}\right) \text {. }
$$

For $r$ and $m$ as above there is a standard homeomorphism $\varphi: E_{m} \rightarrow F_{r}$ which takes $x \rightarrow \Sigma x_{j} r(j)$. Let the inverse of $\varphi$ be called $\psi$. 
We shall frequently write $E$ for $E_{m}, E^{k}$ for $E_{m}^{k}, F$ for $F_{r}$, and $F^{k}$ for $F_{r}^{k}$ when the respective sequences are clear.

Throughout this work $\varepsilon_{j}$ will always denote a quantity that may take on the values 0 or 1 .

1. The symbols $r$ and $m$ shall always denote $\{r(j): j=1,2, \cdots\}$ and $\{m(j): j=1,2, \cdots\}$ respectively. $F_{r}$ and $E_{m}$ will then represent the previously defined sets with $\varphi: E_{m} \rightarrow F_{r}$ and $\psi: F_{r} \rightarrow E_{m}$ the standard homeomorphisms. The maps $\phi$ and $\psi$ induce maps between $N_{1}\left(E_{m}\right)$ and $N_{1}\left(F_{r}\right)$ which we shall again denote by $\varphi$ and $\psi$. The maps have the form

$$
\varphi(\mu)(\{\varphi(x)\})=\mu(\{x\})
$$

for $\mu \in N_{1}(E)$, and

$$
\psi(\mu)(\{\psi(x)\})=\mu(\{x\})
$$

for $\mu \in N_{1}(F)$.

If $x=\left\langle\varepsilon_{1}, \cdots \varepsilon_{k}, 0, \cdots\right\rangle$ is an element of $E_{m}^{k}$ and $\mu \in M\left(E^{k}\right)$ set

$$
a\left(\varepsilon_{1}, \cdots, \varepsilon_{k}\right)=\mu(\{x\}) .
$$

If $y=\sum_{1}^{k} \varepsilon_{j} r(j)$ is an element of $F^{k}$ and $\nu \in M\left(F^{k}\right)$ set

$$
b\left(\varepsilon_{1}, \cdots, \varepsilon_{k}\right)=\nu(\{y\}) \text {. }
$$

We see that

$$
\|\mu\|_{P M}=\sup _{\xi_{1}, \cdots, \xi_{k}}\left|\sum a\left(\varepsilon_{1}, \cdots, \varepsilon_{k}\right) \xi_{1}^{\varepsilon_{1}} \cdots \xi_{k}^{\varepsilon_{k}^{k}}\right|
$$

where $\xi_{j}$ is an arbitrary $m(j)$ root of unity and the sum is taken over all combinations with $\varepsilon_{j}$ being 0 or 1 . Similarly

$$
\|\nu\|_{P M}=\sup _{x}\left|\sum b\left(\varepsilon_{1}, \cdots, \varepsilon_{k}\right) \exp \left(x \sum_{1}^{k} \varepsilon_{j} r(j)\right)\right|
$$

where $x \in R$.

For any $\mu \in N_{1}(E)$ we define

$$
\|\mu\|_{\text {MAX }}=\sup _{\theta_{1}, \cdots \theta_{k}}\left|\sum a\left(\varepsilon_{1} \cdots, \varepsilon_{k}\right) \exp \left(\sum \varepsilon_{j} \theta_{j}\right)\right|
$$

where $\theta_{j} \in R$. Define $\|\boldsymbol{\nu}\|_{\text {MAX }}$ for $\nu \in N_{1}(F)$ by

$$
\|\nu\|_{\text {MAX }}=\sup _{\theta_{1}, \cdots \theta_{k}}\left|\sum b\left(\varepsilon_{1}, \cdots, \varepsilon_{k}\right) \exp \left(\sum \varepsilon_{j} \theta_{j}\right)\right| .
$$


It is clear that $\|\mu\|_{P M} \leqq\|\mu\|_{\text {MAX }}$ and $\|\nu\|_{P M} \leqq\|\nu\|_{\text {MAX }}$. For any standard homeomorphism $\varphi$ we have

$$
\|\varphi \mu\|_{P M} /\|\mu\|_{P M} \leqq\|\mu\|_{\mathrm{MAX}} /\|\mu\|_{P M} .
$$

Similarly

$$
\|\psi \boldsymbol{\nu}\|_{P M} /\|\nu\|_{P M} \leqq\|\nu\|_{M A X} /\|\nu\|_{P M} \text {. }
$$

One should note that if $r$ is a sequence of reals independent mod 1 over the rationals, Kronecher's Theorem [4, p. 99] implies that $\|\boldsymbol{\nu}\|_{\text {MAX }}=\|\nu\|_{P_{M}}$ for $\nu \in N_{1}\left(E_{r}\right)$.

In order to achieve isomorphisms between certain quotient algebras we shall first study the ratios $\|\mu\|_{M A X} /\|\mu\|_{P M}$ and $\|\nu\|_{M+X} /\|\nu\|_{P M}$.

LeMma 1.1. If $\sum(1 / m(j))^{2}<\infty$ then there is a $C$ depending only on $m$ so that $\|\mu\|_{\text {MAX }} /\|\mu\|_{P M} \leqq C$ for all nonzero $\mu \in N_{1}\left(E_{m}\right)$.

Proof. For each $k$, since $M\left(E^{k}\right)$ is finite dimentional, there is a smallest constant $A(k)$ so that $\|\mu\|_{Y A X}\|\mu\|_{P M} \leqq A(k)$ for all nonzero $\mu \in M\left(E^{k}\right)$. We shall show that there are constants $C_{k}$ with $\Pi C_{k}<\infty$ so that $A(k) / A(k-1) \leqq C_{k}$.

The quotient $\|\mu\|_{P M} /\|\mu\|_{M .1 X}$ is equal to

$$
\frac{\sup _{\xi_{j}}\left|\sum_{\varepsilon_{j}}\left[\left(a\left(\varepsilon_{1}, \cdots, \varepsilon_{k-1}, 0\right)+a\left(\varepsilon_{1}, \cdots \varepsilon_{k-1}, 1\right) \xi_{k}\right)\left(\xi_{1}^{\varepsilon_{1}} \cdots \xi_{k-1}^{\varepsilon_{k-1}}\right)\right]\right|}{\sup _{Z_{j}}\left|\sum_{\varepsilon_{j}}\left[\left(a\left(\varepsilon_{1}, \cdots, \varepsilon_{k-1}, 0\right)+a\left(\varepsilon_{1}, \cdots, \varepsilon_{k-1} 1\right) Z_{k}\right)\left(Z_{1}^{\varepsilon_{1}} \cdots Z_{k-1}^{\varepsilon_{k-1}}\right)\right]\right|}
$$

where $\xi_{j}$ are $m(j)$ roots of unity and $Z_{j}$ are complex numbers of modulus 1. By a division and multiplication $\|\mu\|_{P M} /\|\mu\|_{M A X}$ becomes

$$
\begin{aligned}
& \frac{\sup _{\xi_{j}}\left|\sum\left[\left(a(\cdots, 0)+a(\cdots, 1) \xi_{k}\right) \xi_{1}^{\varepsilon_{1}} \cdots \xi_{k-1}^{\varepsilon_{k-1}}\right]\right|}{\sup _{\xi_{k}, Z}\left|\sum\left[\left(a(\cdots, 0)+a(\cdots, 1) \xi_{k}\right) Z_{1}^{\varepsilon_{1}} \cdots Z_{k-1}^{\left.\varepsilon_{k-1}\right]}\right]\right|} \\
& \quad \times \frac{\sup _{\xi_{k}, Z_{j}}\left|\sum\left[\left(a(\cdots, 0)+a(\cdots, 1) \xi_{k}\right) Z_{1}^{\varepsilon_{1}} \cdots Z_{k-1}^{\varepsilon_{k}-1}\right]\right|}{\sup _{Z_{j}}\left|\sum\left[\left(a(\cdots, 0)+a(\cdots, 1) Z_{k}\right) Z_{1}^{\varepsilon_{1}} \cdots Z_{k-1}^{\varepsilon_{k-1}}\right]\right|} .
\end{aligned}
$$

The factor used in division and multiplication in (1.3) is nonzero. If it were zero $\|\mu\|_{P M}$ would be zero and hence $\mu$ would be zero. The fraction on the left of $(1.3)$ is greater than or equal to $1 / A(k-1)$. Choose $z_{j}=y_{j}$ so that the maximum of the denominator in (1.2) is achieved. The fraction on the right in (1.3) is greater than or equal to 


$$
\left|1+\frac{\sum\left[a(\cdots, 1)\left(\xi_{k}-y_{k}\right) y_{1}^{\varepsilon_{1}} \cdots y_{k-1}^{\varepsilon_{k}}\right]}{\sum\left[\left(a(\cdots, 0)+a(\cdots, 1) y_{k}\right) y_{1}^{\varepsilon_{1}} \cdots y_{k-1}^{\varepsilon_{k}-1}\right]}\right| .
$$

If $\sum a(\cdots, 1) y_{1}^{\varepsilon} \cdots y_{k-1}^{s k-1}$ is zero (1.4) is equal to one. Otherwise set $e^{i x}=\xi_{k} / y_{k}$ and (1.4) is equal to

$$
\left|1+\frac{e^{i x}-1}{\left[\frac{\sum\left[a(\cdots, 0) y_{1}^{\varepsilon_{1}} \cdots y_{k-1}^{\varepsilon_{k}-1}\right]}{y_{k} \sum\left[a(\cdots, 1) y_{1}^{\varepsilon_{1}} \cdots y_{k-1}^{\varepsilon_{k}-1}\right]}\right]+1}\right| .
$$

However, in order that the choice $z_{j}=y_{j}$ give $\|\mu\|_{\mathrm{MAX}}$, the quotient

$$
\frac{\sum a(\cdots, 0) y_{1}^{\varepsilon_{1}} \cdots y_{k-1}^{\varepsilon_{k}}{ }^{-1}}{y_{k} \sum a(\cdots, 1) y_{1}^{\varepsilon_{1}} \cdots y_{k-1}^{\varepsilon_{k-1}}}
$$

must be a real positive real number. Call that number $s$ and (1.5) becomes

$$
\left|1+\frac{(\cos x-1)+i \sin x}{s+1}\right|
$$

which is greater than or equal to

$$
1-x^{2} / 2 \text {. }
$$

For an appropriate $\xi_{k},|x|$ is less than or equal to $2 \pi / m(k)$.

From the above calculation we get

$$
\|\mu\|_{P U} /\|\mu\|_{\mathrm{MAX}} \geqq \frac{\left(1-2 \pi^{2} /(m(k))^{2}\right)}{A(k-1)}
$$

and therefore

$$
A(k) \leqq A(k-1) \cdot\left(1+\frac{C^{1}}{(m(k))^{2}}\right)
$$

for some absolute constant $C^{1}$ and for all $m(k)$ sufficiently large. Since $\sum(1 / m(j))^{2}<\infty$ the theorem is proven.

For the symmetric sets $F_{r}$ we shall need the following lemma similar to Lemma 1.1.

Lemma 1.6. Suppose that $\sum(r(j+1) / r(j))^{2}<1 / 24$. Choose a real number $x_{0}$ and define the interval $I$ to be

$$
\left\{x:\left|x-x_{0}\right|<2\left(\sum_{1}^{k} 1 / r(j)\right)\right\} .
$$

There is then a constant $C_{1}$ independent of $k$ and $x_{0}$, so that

$$
\|\nu\|_{\text {MAX }} / \sup |\hat{\nu}(x)|<C_{1} \text {, for all nonzero } \nu \in M\left(F_{r}^{k}\right) \text {. }
$$


Proof. Fix $k$ and choose a nonzero $\nu \in M\left(F_{r}^{k}\right)$. There exists real numbers $\theta_{1}, \cdots, \theta_{k}$ less than or equal to one, for which

$$
\|\nu\|_{\text {MAX }}=\left|\sum b\left(\varepsilon_{1}, \cdots, \varepsilon_{k}\right) \exp \left(\sum \varepsilon_{j} \theta_{j}\right)\right| \text {. }
$$

Define the functions $\hat{\nu}_{k}, \cdots, \hat{\nu}_{2}, \hat{\nu}_{1}=\hat{\nu}$ on $R$ by

$$
\hat{\nu}_{j}(\mathrm{x})=\sum\left[b\left(\varepsilon_{1}, \cdots, \varepsilon_{k}\right) \exp \left(\sum_{1}^{j-1} \varepsilon_{j} \theta_{j}\right) \exp x\left(\sum_{j}^{k} \varepsilon_{j} r(j)\right)\right] .
$$

Let us estimate $\sup _{x \in I_{1}}\left|\hat{\nu}_{k-1}(x)\right| /|| \nu \|_{\text {MAx }}$ where

$$
I_{1}=\left\{x:\left|x-x_{0}\right| \leqq \sum_{k-1}^{k}(2 / r(j))\right\} .
$$

There is an $x_{0}^{\prime}$ within $(1 / r(k))$ of $x_{0}$ for which $x_{0}^{\prime} \cdot r(k)=\theta_{k}(\bmod 1)$. Pick $x_{1}$ within $1 / r(k-1)$ of $x_{0}^{\prime}$ so that $x_{1} \cdot r(k-1)=\theta_{k-1}(\bmod 1)$. Then

$$
\sup _{x \in I_{1}}\left|\hat{\nu}_{k-1}(x)\right| /|| \nu\left\|_{\text {MAX }} \geqq\left|\hat{\nu}_{k-1}\left(x_{1}\right)\right| /|| \nu\right\|_{\operatorname{MAX}} \cdot
$$

As a function of $x, \hat{\nu}_{k}(x)$ is the Fourier Stieltjes transform of a measure $\nu_{k}$ having support in $[0, r(k)]$. Now,

$$
\begin{aligned}
\left|\hat{\nu}_{k-1}\left(x_{1}\right)\right| /|| \nu \|_{\text {MAX }} & =\left|\hat{\nu}_{k}\left(x_{1}\right)\right| /\left|\hat{\nu}_{k}\left(x_{0}^{\prime}\right)\right| \\
& =\left|1+\frac{\hat{\nu}_{k}^{\prime}\left(x_{0}^{\prime}\right)}{\hat{\nu}_{k}\left(x_{0}^{\prime}\right)}\left(x_{1}-x_{0}^{\prime}\right)+\frac{\hat{\nu}_{k}^{\prime \prime}\left(x_{0}^{\prime}\right)}{\hat{\nu}_{k}\left(x_{0}^{\prime}\right)} \frac{\left(x_{1}-x_{0}^{\prime}\right)^{2}}{2}+\cdots\right|
\end{aligned}
$$

$\left|\hat{\nu}_{k}\right|^{2}$ has a maximum at $x_{0}^{\prime}$. Therefore, if $\hat{\nu}_{k}=f+i g$, with $f$ and $g$ real, $f \cdot f^{\prime}+g \cdot g^{\prime}=0$ at $x_{0}^{\prime}$. But, at $x_{0}^{\prime}$,

$$
\begin{aligned}
\hat{\nu}_{k}^{\prime} / \hat{\nu}_{k} & =f^{\prime}+i g^{\prime} / f+i g \\
& =\left(f f^{\prime}+g g^{\prime}+i\left(f g^{\prime}-f^{\prime} g\right)\right) / f^{2}+g^{2},
\end{aligned}
$$

which is purely imaginary. Therefore,

$$
\left|\widehat{\nu}_{k-1}\left(x_{1}\right)\right| /|| \nu \|_{\operatorname{MAx}} \geqq 1-\left|\frac{\hat{\nu}_{k}^{\prime \prime}\left(x_{0}^{\prime}\right)}{\widehat{\nu}_{k}\left(x_{0}^{\prime}\right)} \frac{\left(x_{1}-x_{0}^{\prime}\right)^{2}}{2}+\cdots\right| .
$$

If a measure $\mu$ has support in $[0, \delta]$ a theorem of Bernstein [1, p. 138] shows that for all $x$

$$
\left[\widehat{\mu}^{\prime}(x) \mid \leqq \delta\|\mu\|_{P M}\right.
$$

and hence its $n t h$ derivative $\hat{\mu}^{(n)}$ has

$$
\left|\hat{\mu}^{(n)}(x)\right| \leqq \delta^{n}\|\mu\|_{P M} .
$$

Since $\nu_{k}$ has support in $[0, r(k)]$ we obtain

$$
\left|\hat{\nu}_{k-1}\left(x_{1}\right)\right| /\|\mid \nu\|_{\text {MAX }} \geqq 1-\left(r(k)^{2} / r(k-1)^{2}\right) .
$$


In effect, we have just shown that there is an $x_{1} \in I_{1}$ for which

$$
\|\nu\|_{\text {MLX }} /\left|\hat{\nu}_{k-1}\left(x_{1}\right)\right| \leqq 1+2(r(k) / r(k-1))^{2} .
$$

Assume that for some $j<k-1$ there is an

$$
x_{j} \in I_{j}=\left\{x:\left|x-x_{0}\right| \leqq \sum_{k=j}^{k}(2 / r(l))\right\}
$$

for which

$$
\|\nu\|_{\text {MAX }} /\left|\hat{\nu}_{k-j}\left(x_{j}\right)\right| \leqq \prod_{l=k-j}^{\infty}\left(1+24(r(l+1) / r(l))^{2}\right) .
$$

We shall show there is then an $x_{j+1} \in I_{j+1}$ for which

$$
\begin{aligned}
& \|\boldsymbol{\nu}\|_{\mathrm{M} . \mathrm{XX}} /\left|\hat{\nu}_{k-(j+1)}\left(x_{j+1}\right)\right| \\
& \quad \leqq \prod_{l=l i-j-1}^{\infty}\left(1+24(r(l+1) / r(l))^{2}\right) .
\end{aligned}
$$

Consider $S=\left\{x:\left|x-x_{j}\right| \leqq 1 / r(k-(j+1))\right\}$. If $\left|\hat{\nu}_{k-j}\right|$ does not have a relative maximum in $S$ greater than or equal to $\left|\hat{\nu}_{k-j}\left(x_{j}\right)\right|$, then $\left|\hat{\nu}_{k-j}\right|$ would be greater than or equal to $\left|\hat{\nu}_{k-j}\left(x_{j}\right)\right|$ on some interval in $S$ of length equal to $1 / r(k-(j+1))$. However there would be an $x_{j+1}$ in the interval for which $x_{j+1} \cdot r(k-(j+1))=\theta_{k-(j+1)}(\bmod 1)$ and hence $\hat{\nu}_{k-(j+1)}\left(x_{j+1}\right)=\hat{\nu}_{k-j}\left(x_{j+1}\right)$, which implies the induction step. Let us assume therefore that there is an $x_{j}^{\prime}$ where

$$
\left|x_{j}^{\prime}-x_{0}\right| \leqq\left(1 / r(k-(j+1))+\sum_{k-j}^{k} 2 / r(l)\right),
$$

$\left|\hat{\nu}_{k-j}\left(x_{j}^{\prime}\right)\right| \geqq\left|\hat{\nu}_{k-j}\left(x_{j}\right)\right|$ and at which $\left|\hat{\nu}_{k-j}\right|$ has a relative maximum. As before, choosing $x_{j+1}$ within $1 / r(k-j+1)$ of $x_{j}^{\prime}$ and satisfying $x_{j+1} \cdot r(k-(j+1))=\theta_{k-(j+1)}$ gives

$$
\begin{aligned}
& \left|\hat{\nu}_{k-(j+1)}\left(x_{j+1}\right) / \hat{\nu}_{k-j}\left(x_{j}^{\prime}\right)\right|=\left|\hat{\nu}_{k-j}\left(x_{j+1}\right) / \hat{\nu}_{k-j}\left(x_{j}^{\prime}\right)\right| \\
& \quad \geqq \sum 1-\left|\frac{\hat{\nu}_{k-j}^{\prime \prime}\left(x_{j}^{\prime}\right)}{\hat{\nu}_{k-j}\left(x_{j}^{\prime}\right)} \cdot \frac{\left(x_{j+1}-x_{j}^{\prime}\right)^{2}}{2}+\cdots\right| .
\end{aligned}
$$

$\hat{\nu}_{k-j}$ as a function of $x$ is the Fourier Stieltjes of a measure $\nu_{k-j}$ having support in $[0,2 r(k-j)]$. Since $\left\|\boldsymbol{\nu}_{k-j}\right\|_{P M} \leqq\|\nu\|_{\mathrm{MAX}}$, the previously stated theorem of Bernstein gives

$$
\left|\hat{\nu}_{k-j}^{(n)}\left(x^{\prime}\right)\right| \leqq(2 r(k-j))^{n}\|\nu\|_{\operatorname{MAX}} .
$$

However

$$
\begin{aligned}
\|\nu\|_{\text {MAX }} & \leqq\left[\prod_{l=k-j}^{\infty}\left(1+24(r(l+1) / r(l))^{2}\right)\right] \times\left|\hat{\nu}_{k-j}\left(x_{j}^{\prime}\right)\right| \\
& \leqq e^{24 \Sigma(r(l+1) / r(l))^{2}} \cdot\left|\hat{\nu}_{k-j}\left(x_{j}^{\prime}\right)\right| \\
& \leqq 3\left|\hat{\nu}_{k-j}\left(x_{j}^{\prime}\right)\right|
\end{aligned}
$$


Since $\Sigma(r(l+1) / r(l))^{2} \leqq(1 / 24)$. Therefore in $(1.8)$,

$$
\left|\hat{\nu}_{(k-j+1)}\left(x_{j+1}\right) / \hat{\nu}_{k-j}\left(x_{j}^{\prime}\right)\right| \geqq 1-12\left(r(k-j) / r(k-(i+1))^{2}\right.
$$

and hence (1.7) is true, finishing the induction.

Lemma 1.6 in its present form is an adaptation and extension of a lemma of Meyer [12]. Previously we had much more stringent conditions on the $r$, to arrive at a similar conclusion to Lemma 1.6.

To utilize the Lemmas 1.1 and 1.6 to obtain isomorphisms of restriction algebras we shall introduce some functional analysis.

Let $V$ represent a Banach Space and $V^{*}$ its dual. For $r>0$ let $B_{r}=\left\{t: t \in V^{*},\|t\| \leqq r\right\}$. A set $O \subseteq V^{*}$ is said to be open in the bounded topology on $V^{*}$ if and only if $O \cap B_{r}$ is open in the relative weak* topology of $B_{r}$ for all $\mathrm{r}>0$. For a distribution of the bounded topology the reader should consult [6, p. 427].

Lemma 1.10. Let $V, W$ be Banach spaces with duals $V^{*}$ and $W^{*}$. Let $K \subset V^{*}$ be a weak* dense subspace of $V^{*}$. Suppose that $T: K \rightarrow W$ is linear and continuous when $K$ has the topology induced by the bounded topology on $V^{*}$ and $W^{*}$ has the weak topology. Then there exists a bounded linear transformation $S: W \rightarrow V$ for which $T=S^{*} / K$.

Proof. For each $w \in W$, define the linear functional $T_{w}$ on $K$ by

$$
T_{w}(t)=T t(w) \text {. }
$$

Each $T_{w}$ is continuous in the topology induced by the bounded topology of $V^{*}$ which is a locally convex topology by Corollary 5, page 428 of [6]. Hence by the Hahn-Banach theorem there exists an extension $\widetilde{T}_{w}$ of $T_{w}$ to all of $V^{*}$, continuous in the bounded topology of $V^{*}$.

By Theorem 6, page 428 of [6], $\widetilde{T}_{w}$ is continuous in the weak* topology on $V^{*}$. Hence there exists an element $v \in V$ such that $T_{w}(t)=t(v)$ for all $t \in K$. Since $K$ is assumed weak* dense in $V^{*}$, the element $v$ is determined by $w$. Define $S: W \rightarrow V$ by $S(w)=v$. $S$ is linear. Since $K$ is weak* dense $S$ is closed. Therefore by the Closed Graph Theorem $S$ is bouned. If $t \in K, w \in W$

$$
S^{*} t(w)=t(S(w))=T t(w),
$$

which completes the proof.

It is clear that $N_{1}\left(E_{m}\right)$ and $N_{1}\left(F_{r}\right)$ are weak ${ }^{*}$ dense in $N\left(E_{m}\right)$ and $N\left(F_{r}\right)$, respectively. By studying the continuity of the standard maps between $N_{1}\left(E_{m}\right)$ and $N_{1}\left(F_{r}\right)$, we shall be able to use Lemma 1.10 to 
obtain isomorphisms between $A\left(E_{m}\right)$ and $A\left(F_{r}\right)$ for certain classes of sequences $m$ and $r$.

Choose $\mu \in N_{1}(E)$. For each $k$ we define an approximating measure $\mu_{k}$ in $M\left(E^{k}\right)$ by

$$
\mu_{k}(\{x\})=\sum_{y \in D} \mu(\{y\})
$$

where $x \in E^{k}$ and $D=\left\{y: y \in E\right.$ and $y_{j}=x_{j}$ for $\left.j \leqq k\right\}$. Let

$$
\Gamma^{k}=\left\{\gamma: \gamma \in \Sigma Z(m(j)) \text { and } \gamma_{j}=0 \text { if } j>k\right\} .
$$

If $\gamma \in \Gamma^{k} \hat{\mu}_{k}(\gamma)=\hat{\mu}(\gamma)$. It is easy to see that

$$
\left\|\mu_{k}\right\|_{P M}=\sup _{\gamma \in \Gamma}\left|\hat{\mu}_{k}(\gamma)\right| \text {. }
$$

To each $\lambda \in M\left(E^{k}\right)$ we associate the measure $\lambda^{\prime}$ in $M\left(E^{k}\right)$ defined by

$$
\lambda^{\prime}(\{x\})=\left\{\begin{array}{lll}
0 & \text { if } & x_{k}=0 \\
\lambda(\{x\}) & \text { if } & x_{k}=1
\end{array} .\right.
$$

It is not hard to see that

$$
\left\|\lambda^{\prime}\right\|_{P M} \leqq 2\|\lambda\|_{P, M} \cdot
$$

Choose $\nu \in N_{1}(F)$. For each $k$ define an approximating measure $\nu_{k}$ in $M\left(F^{k}\right)$ by

$$
\nu_{k}(\{x\})=\sum_{y \in D} \nu(\{y\})
$$

where $x=\sum_{1}^{k} x_{j} r(j)$ and $D=\left\{y: y=\Sigma \varepsilon_{j} r(j)\right.$ and $\varepsilon_{j}=x_{j}$ for $\left.j \leqq k\right\}$.

To each $\beta \in M\left(F^{k}\right)$ we associate the measure $\beta^{\prime}$ in $M\left(F^{k}\right)$ defined by

$$
\beta^{\prime}(\{x\})=\left\{\begin{array}{lllll}
0 & \text { if } & x=\sum_{1}^{k} \varepsilon_{j} r(j) & \text { and } & \varepsilon_{k}=0 \\
1 & \text { if } & x=\sum_{1}^{k} e_{j} r(j) & \text { and } & \varepsilon_{k}=1
\end{array} .\right.
$$

We are now ready to prove the following theorem.

TheOREM 1.11. If $\Sigma(1 / m(j))^{2}<\infty$ and $\Sigma(r(j+1) / r(j))^{2}<\infty$ then $A\left(E_{m}\right)$ is isomorphic to $A\left(F_{r}\right)$.

We shall break the proof into two lemmas.

Lemma $A$. Let $F_{r}$ be any symmetric set. Let $\Sigma(1 / m(j))^{2}<\infty$ $\varphi: E_{m} \rightarrow F_{r}$ the standard homeomorphism. Then there is an iso- 
morphism into $\Phi: A\left(F_{r}\right) \rightarrow A\left(E_{m}\right)$ given by

$$
\Phi(f)=f \circ \varphi, \quad f \in A\left(F_{r}\right) .
$$

Proof. We shall study the continuity properties of

$$
\varphi: N_{1}(E) \rightarrow N_{1}(F) \text {. }
$$

For $f \in A(F)$ define

$$
U_{\varepsilon, f}=\left\{\nu: \nu \in N_{1}(F) \text { and }|(\nu, f)|<\varepsilon\right\} .
$$

To establish that $\varphi$ is continuous from the bounded weak* topology of $N_{1}(E)$ to the weak* topology of $N_{1}(F)$ it is sufficient to prove that the zero element of $N_{1}(E)$ is an interior point of $\varphi^{-1}\left(U_{\varepsilon, f}\right)$ (i.e., that $\varphi$ is continuous at 0 ). This follows at once if we prove that given $a$ and $\varepsilon$, there exists $\delta, k$ such that if for $\mu \in N_{1}(E)$

$$
\begin{aligned}
& \|\mu\|_{P M} \leqq a \text { and }|\hat{\mu}(\gamma)|<\delta \text { for } \gamma \in \Gamma^{k} \\
& \varphi(\mu) \text { is an element of } U_{\varepsilon, f} .
\end{aligned}
$$

In view of Lemma 1.1 (1.12) follows if we can show that given $a, \varepsilon$, and $M$ then there exists $\delta, k$ such that for $\mu \in N_{1}(E)$,

$$
\|\mu\|_{P M} \leqq a \text { and } \hat{\mu}(\gamma)<\delta \text { for } \gamma \in \Gamma^{k}
$$

then

$$
|\widehat{\varphi(\mu)}(x)|<\varepsilon \text { for }|x| \leqq M \text {. }
$$

We first estimate $\left|\widehat{\phi(\mu)}-\widehat{\phi\left(\mu_{k}\right)}\right|$ for $\mu \in M\left(E^{s}\right)$.

$$
\begin{aligned}
& \left.\left|\widehat{\varphi(\mu)}(x)-\widehat{\varphi\left(\mu_{k}\right)}(x)\right| \leqq \sum_{k}^{s-1} \mid \widehat{\varphi\left(\mu_{j+1}\right)}\right)(x)-\widehat{\varphi\left(\mu_{j}\right)}(x) \mid \\
& \leqq \sum_{k}^{s-1}|\exp (-x r(j+1))-1| \cdot\left\|\varphi\left(\mu_{j+1}^{\prime}\right)\right\|_{P M} .
\end{aligned}
$$

By Lemma 1.1, for any $s$

$$
\left|\widehat{\varphi(\mu)}(x)-\widehat{\varphi\left(\mu_{k}\right)}(x)\right| \leqq 4 \pi C|x|\|\mu\|_{P M} \cdot \sum_{k+1}^{\infty} r(j) \text {. }
$$

For $\mu$ with $\|\mu\|_{P M} \leqq a$, pick $\delta<\varepsilon / 2 C$ where $C$ is the constant of Lemma 1.1 and choose $k$ so that $4 \pi C M a \sum_{k+1}^{\infty} r(j)<\varepsilon / 2$. If $|\hat{\mu}(\gamma)|<\delta$ for $\gamma \in \Gamma^{k}$, then $\left\|\mu_{k}\right\|_{P M}<\delta$ and by Lemma $1.1\left\|\varphi\left(\mu_{k}\right)\right\|_{P M}<\varepsilon / 2$. If $|x| \leqq M$, then $\left|\widehat{\varphi(\mu)}(x)-\widehat{\phi\left(\mu_{k}\right)}(x)\right|<\varepsilon / 2$ so

$$
|\widehat{\varphi}(\mu)(x)|<\varepsilon, \text { for }|x| \leqq M .
$$

The conditions of Lemma 1.10 are satisfied so $\varphi=\Phi^{*}$ for some 
linear $\Phi: A(F) \rightarrow A(E)$. For $\mu \in N_{1}(E)$ and $f \in A(F)$

$$
(\Phi f, \mu)=(f, \varphi(\mu)) \text {. }
$$

Therefore if $x \in \bigcup_{1}^{\infty} E^{s}$

$$
\Phi f(x)=f(\varphi(x)) \text {. }
$$

Since $\varphi, f$ and $\Phi f$ are continuous, $\Phi$ is the linear map wanted.

LEMma $B$. Let $F_{r}$ be a symmetric set with $\Sigma(r(j+1) / r(j))^{2}<\infty$. Let $\psi: F_{r} \rightarrow E_{m}$ be the standard homeomorphism of $F_{r}$ with some $E_{m}$. Then there is an isomorphism into $\bar{\Psi}: A\left(E_{m}\right) \rightarrow A\left(F_{r}\right)$ given by

$$
\bar{\Psi}(f)=f \circ \psi, \quad f \in A\left(E_{m}\right) .
$$

Proof. There is an $l$ so that $\sum_{l+1}^{\infty}(r(j+1) / r(j))^{2}<1 / 24 . \quad F$ is a union of $2^{l}$ sets which are translations of the set $F^{\prime}=\{x: x=$ $\left.\sum_{l+1}^{\infty} \varepsilon_{j} r(j)\right\}$. It is therefore sufficient to prove the theorem for $F^{\prime}$. For convenience, assume $F_{r}$ has the property $\sum_{1}^{\infty}(r(j+1) / r(j))^{2}<1 / 24$. We shall show as in Lemma A that $\psi: N_{1}\left(F_{r}\right) \rightarrow N_{1}\left(E_{m}\right)$ has the required continuity properties to be the adjoint of a continuous linear map $\bar{\Psi}: A\left(E_{m}\right) \rightarrow A\left(F_{r}\right)$ satisfying $\bar{\Psi}(f)=f \circ \psi$.

Using Lemmas 1.6 and 1.10 as in Lemma $A$, it is enough to show that if $a, \varepsilon, M$ are given, then there exists $\delta, x_{1}, \cdots, x_{t}$ so that the following holds.

If $\nu \in N_{1}(F),\|\nu\|_{P M} \leqq a$ and $\hat{\nu}\left(x_{j}\right)<\delta$ for $j=1, \cdots, t$, then $|\widehat{\psi(\nu)}(\gamma)|<\varepsilon$ for $\gamma \in \Gamma^{M}$.

Choosing $\nu \in N_{1}(F)$ with $\|\nu\|_{P M} \leqq a$ and estimating $\left|\hat{\nu}-\hat{\nu}_{k}\right|$ gives

$$
\begin{aligned}
\left|\hat{\nu}(x)-\hat{\nu}_{k}(x)\right| & \leqq \sum_{k}^{S}\left|\hat{\nu}_{j+1}(x)-\hat{\nu}_{j}(x)\right| \\
& \leqq \sum_{k}^{\infty}|\exp (-x r(j+1))-1|\left\|\nu_{j+1}^{\prime}\right\|_{P M} .
\end{aligned}
$$

Lemma 1.1 and 1.6 show that the $P M$ norm on $N_{1}\left(F_{r}\right)$ and $N_{1}\left(E_{m^{\prime}}\right)$ are equivalent when $\sum\left(1 / m^{\prime}(j)\right)^{2}<\infty$. Hence

$$
\begin{aligned}
\left|\hat{\nu}(x)-\hat{\nu}_{k}(x)\right| & \leqq 4 \pi x C_{1} C\|\nu\|_{P M} \sum_{k+1}^{\infty} r(j) \\
& \leqq 8 \pi|x| C_{1} C a \cdot r(k+1) .
\end{aligned}
$$

An easy consequence of the condition $\Sigma(r(j+1) / r(j))^{2}<1 / 24$ is that

$$
\lim _{k \rightarrow \infty} 8 \pi C_{1} \cdot C . a \cdot\left(\sum_{1}^{k} 2 / r(j)\right) \cdot r(k+1)=0 .
$$


Pick $k \geqq M$ large enough so that

$$
8 \pi C_{1} C a\left(\sum_{1}^{k} 2 / r(j)\right) r(k+1)<\varepsilon / 4 C_{1} .
$$

Then

$$
\left|\hat{\nu}(x)-\hat{\nu}_{k}(x)\right|<\varepsilon / 4 C_{1}
$$

for $|x|<\sum_{1}^{k}(2 / r(j))$. By Lemma 1.6 there is an $\mathrm{x}_{0}$ with

$$
\left|x_{0}\right|<\sum_{1}^{k}(2 / r(j)
$$

so that for $\nu_{k} \in M\left(F^{k}\right)$

$$
\left\|\nu_{k}\right\|_{\text {MAX }} /\left\|\hat{\nu}_{k}\left(x_{0}\right)\right\|<C_{1} \text {. }
$$

By a theorem of Bernstein [1, p. 138]

$$
\left|\hat{\nu}_{k}\left(x_{0}\right)-\hat{\nu}_{k}\left(x_{*}\right)\right| \leqq C_{1}\left|\hat{\nu}_{k}\left(x_{0}\right)\right|\left(\sum_{1}^{\infty} r(j)\right)\left|x_{*}-x_{0}\right| .
$$

Therefore, if $\left|x_{*}-x_{0}\right|<1 / 2\left(\sum r(j)\right) \cdot C_{1}$

$$
\left\|\nu_{k}\right\|_{\text {MAX }} /\left|\hat{\nu}_{k}\left(x_{*}\right)\right| \leqq 2 C_{1} \text {. }
$$

Choose for $i=1, \cdots, t ; x_{i}$ with $\left|x_{i}\right| \leqq \sum_{1}^{k}(2 / r(j))$ so that for every $x$ with $|x| \leqq \sum_{1}^{k}(2 / r(j))$ there is an $x_{j}$ with $\left|x-x_{j}\right|<1 / 2(\Sigma r(j)) \cdot C_{1}$. If $\left|\hat{\nu}\left(x_{j}\right)\right|<\varepsilon / 4 C_{1}$ for $\mathrm{x}_{j}, j=1, \cdots, t$, then $\left|\hat{\nu}_{k}\left(x_{j}\right)\right|<\varepsilon / 2 C_{1}$ by (1.14), and by (1.15) $\left\|\nu_{k}\right\|_{\text {MAX }}<\varepsilon$. Consequently, $\left\|\psi\left(\nu_{k}\right)\right\|_{P M}<\varepsilon$. Since $k>M$ we see that $|\widehat{\psi(\nu)}(\gamma)|<\varepsilon$ for $\gamma \in \Gamma^{u}$.

As in Lemma A, the continuity conditions of Lemma 1.10 are satisfied and

$$
\bar{\Psi}(f)=f \circ \psi \cdot
$$

Theorem 1.11 is an immediate consequence of Lemmas $\mathrm{A}$ and $\mathrm{B}$. Meyer [12] has proven that if $\Sigma(r(j+1) / r(j))<\infty$ and

$$
\Sigma(s(j+1) / s(j))<\infty
$$

then $A\left(F_{r}\right) \cong A\left(F_{s}\right)$. Lemmas 1.6 was an analogue and improvement on his main lemma which allowed us to obtain the theorem with square summability.

If $r_{0}(j)=\left\{e^{-j} \cdot 2^{-j^{2}}\right\}$ then every $A\left(F_{r}\right)$ and $A\left(E_{m}\right)$ with

$$
\Sigma(r(j+1) / r(j))^{2}<\infty \text { and } \Sigma(1 / m(j))^{2}<\infty
$$

is isomorphic to $A\left(F_{r_{0}}\right)$. The isomorphisms are given by

$$
f \rightarrow f \circ \varnothing
$$


where $f$ is in an appropriate restriction algebra and $\varphi$ one of the standard homeomorphisms. We shall call an isomorphism between any two restriction algebras induced in this manner a standard isomorphism. If $A\left(F_{r}\right)$ or $A\left(E_{m}\right)$ is isomorphic to $A\left(F_{r_{0}}\right)$ by standard isomorphisms, $F_{r}$ or $E_{m}$ will then be said to belong to the class $M_{y}$. One should note that for $\mu \in N_{1}\left(F_{r_{0}}\right),\|\mu\|_{P M}=\|\mu\|_{M A X}$.

Define sets of multiplicity and uniqueness as in [7, p. 52]. In [7, p. 100] it is shown that if $\alpha \in[0,1 / 2)$ one can construct sets $F_{r}$ of multiplicity with $r(j+1) / r(j)=0\left(j^{-\alpha}\right)$. The next theorem shows, in particular, that if $r(j+1) / r(j)=0\left(j^{-\alpha}\right)$ with $\alpha \in(1 / 2, \infty)$ then $F_{r}$ is a set of uniqueness.

Theorem 1.16. Suppose that $\Sigma(r(j+1) / r(j))^{2}<\infty$. Then $F_{r}$ is $a$ set of synthesis and there is a constant $B$ so that for all $S \in N\left(F_{r}\right)$

$$
\|S\|_{P M} \leqq B \varlimsup \lim |\hat{S}(x)| \text {. }
$$

Hence $F_{r}$ is a set of uniqueness.

Proof. Choose $l$ so that $\sum_{l+1}^{\infty}(r(j+1) r(j))^{2}<1 / 24$. Then $F$ is a union of $2^{l}$ disjoint sets of the form $a(\varepsilon)+F(l)$ where $\varepsilon=\left\langle\varepsilon_{1}, \cdots, \varepsilon_{l}\right\rangle$ and $F(l)=\left\{x: x=\sum_{l+1}^{\infty} \varepsilon_{j} r(j)\right\}$. We can find $2^{l}$ functions $\varphi_{\varepsilon}$ in $A(R)$ where $\varphi_{s}=1$ on $a(\varepsilon)+F(l)$ and 0 on the other sets. Let $S \in P M$ with support in $F_{r} . \quad S=\Sigma_{\varepsilon} \varphi_{s} S$ and hence if $\varphi_{\varepsilon} S \in N(a(\varepsilon)+F(l))$ for each $\varepsilon, S \in N\left(F_{r}\right)$. Moreover, for some $\varepsilon$ the inequality

$$
\left\|\varphi_{\varepsilon} S\right\|_{P M} \geqq 2^{-l}\|S\|_{P M}
$$

must hold. If $\|S\|_{P M}>B \lim |\widehat{S}(x)|$ we see that

$$
\left\|\varphi_{\varepsilon} S\right\|_{P M} \geqq \frac{2^{-l} B}{\left\|\varphi_{\varepsilon}\right\|_{A}} \varlimsup|\widehat{\lim }| \widehat{\varphi_{\varepsilon}} S(x) \mid \text {. }
$$

We may therefore assume that $\Sigma(r(j+1) / r(j))^{2}<1 / 24$.

Lemma 1.6 and [12, Proposition 2.2.3] imply that there is a natural isomorphism $T$ from $A\left(F_{r}^{k} \times[-2 r(k+1), 2 r(k+1)]\right)$ in $A(R \times R)$ to $A\left(F_{r}^{k}+[-2 r(k+1), 2 r(k+1)]\right.$ with norm

$$
T \leqq\left(1-\alpha 4 r(k+1) \cdot\left(\sum_{1}^{k} 1 / r(j)\right)\right)^{-1}
$$

and $\left\|T^{-1}\right\|=1$, where $\alpha \leqq 1$ and is independent of $k$. For large enough $k$ the norm is smaller than some constant $B_{1}$. For each $x \in R$ consider the funtion $f_{x} \in A\left(F_{r}^{k}+[-2 r(k+1), 2 r(k+1)]\right)$

$$
f_{x}(y)=\exp (x y)-\exp \left(x \cdot \Sigma_{1}^{k} \varepsilon_{j} r(j)\right) \quad \text { for }\left|y-\Sigma_{1}^{k} \varepsilon_{j} r(j)\right| \leqq 2 r(k+1) \text {. }
$$

Its image in $A\left(F_{r}^{k} \times[-2 r(k+1), 2 r(k+1)]\right.$ is 


$$
\widetilde{f}_{x}(t, y)=\exp (x t) \cdot(\exp (x y)-1) \text {. }
$$

Then

$$
\left\|f_{x}\right\|_{A\left(F_{r}^{k}+[\cdot]\right)} \leqq B_{1}\left\|\tilde{f}_{x}\right\|_{A\left(F_{r}^{k} \times[\cdot]\right)} \leqq B_{2}|x| r(k+1)
$$

Define $v_{k} \in M\left(F_{r}^{k}\right)$ by

$$
v_{k}\left(\left\{\Sigma \varepsilon_{j} r(j)\right\}\right)=\left(\widehat{\left.\left.S\right|_{\Sigma_{1}^{k} \varepsilon_{j} r(j)+[\cdot]}\right)(0)} .\right.
$$

where $S$ is a given element of $P M$ with support in $F_{r}$. Then for sufficiently large $k$

$$
\left|\hat{S}(x)-\widehat{v}_{k}(x)\right|=\left|\left(S, f_{x}\right)\right| \leqq B_{2} \cdot|x| \cdot\|S\|_{P M} \cdot r(k+1) .
$$

By Lemma 1.6 we have that

$$
\widehat{v}_{k}(x) \rightarrow \widehat{S}(x) \forall x \in R ; \lim \left\|v_{k}\right\|_{P M} \leqq C\|S\|_{P M}
$$

and hence $S \in N\left(F_{r}\right)$ and $F_{r}$ is a set of synthesis.

For convenience assume that $\|S\|_{P M}=1$ and $|\hat{S}(0)|>1 / 2$. Suppose that $|\hat{S}(x)|<\varepsilon$ for $x>x_{0}$. Pick a constant $k_{0}$ so that

$$
\left(x_{0}+4 \cdot \Sigma_{1}^{k} r(j)\right) B_{2}\|S\|_{P M} \cdot r(k+1)<\varepsilon
$$

for $k>k_{0}$. Then if $k>k_{0}$

$$
\left|\widehat{v}_{k}(x)\right|<2 \varepsilon
$$

for all $x$ satisfying $\left|x-x_{*}\right| \leqq \Sigma_{1}^{k}(2 / r(j))$ where $x_{*}$ is the center of the interval $\left[x_{0}, x_{0}+4 \sum_{1}^{k}(1 / r(j))\right]$. Since $\left|\hat{v}_{k}(0)\right|>1 / 2$ Lemma 1.6 shows that

$$
\varepsilon>1 / 4 C_{1}
$$

Theorem 1.16 is essentially methods of McGehee and Meyer utilizing Lemma 1.6.

We next examine the sets $E_{m}$. By $[15, \mathrm{p} .166]$ they are sets of synthesis. If $m(j)=2$ for all but a finite number of $j, E_{m}$ has positive measure and there is an $S \in N\left(E_{m}\right)$ with $\inf _{T} \sup _{\gamma \in \sim T}|\hat{S}(\gamma)|=0$. The following is a converse.

THEOREM 1.17. Let $m(j)$ be a sequence of integers with infinitely many $m(j) \geqq 3$. Then there is a constant $C$ so that for all $S \in N\left(E_{m}\right)$

$$
\|S\|_{P M} \leqq C \inf _{T} \sup _{\gamma \in \sim T}|\hat{S}(\gamma)|
$$

where $T$ is any finite set in $\Sigma Z_{m(j)}$. 
Proof. Let $S \in N(E)$ and assume for simplicity that $\|S\|_{P M}=1$ and $\hat{S}(0)>3 / 4$. Let $\left\{\mu_{k}\right\}$ be the measure defined by

$$
\mu_{k}\{x\}=\left({\widehat{S \mid \prod_{\substack{\prod_{j=k+1}^{\infty} \\ j=(j)}}^{\infty} z_{m}}}\right)(0)
$$

where $x=\left\langle\varepsilon_{1}, \cdots, \varepsilon_{k}, 0,0, \cdots\right\rangle$. Let $\gamma^{s} \varepsilon \sum \Gamma_{m(j)}$ be that element with

$$
\gamma_{j}^{s}=\left\{\begin{array}{lll}
0 & \text { if } & j \neq s \\
1 & \text { if } & j=s
\end{array} .\right.
$$

Then for $1 \leqq s \leqq k$

$$
\begin{aligned}
\hat{\mu}_{k}\left(\gamma^{s}\right)= & \sum_{\varepsilon(s)=0} a(\varepsilon(1), \cdots, \varepsilon(k)) \\
& +\sum_{\varepsilon(s)=1} a(\varepsilon(1), \cdots, \varepsilon(k)) \exp (1 / m(s)) .
\end{aligned}
$$

If we call $\sum_{\varepsilon(s)=0} a(\varepsilon(1), \cdots, \varepsilon(k))=\alpha$

$$
\sum_{\varepsilon(s)=1} a(\varepsilon(1), \cdots, \varepsilon(k))=\beta \quad \text { then } \hat{\mu}_{k}(0)=\alpha+\beta .
$$

It is easy to see that $\alpha \leqq 1$ and $\beta \leqq 2$. Therefore

$$
\begin{aligned}
\left|\hat{\mu}_{k}\left(\gamma^{s}\right)-\hat{\mu}_{k}(0)\right| & \leqq 2|\exp (1 / m(s)-1)| \\
& \leqq 4 \pi / m(s) .
\end{aligned}
$$

Therefore, if $m(s)>8 \pi$

$$
\left|\hat{\mu}_{k}\left(\gamma^{s}\right)\right|>1 / 4 \text {. }
$$

Let $\tilde{\gamma}^{s} \in \Sigma \Gamma_{m(j)}$ be the element with

$$
\tilde{\gamma}_{j}^{s}=\left\{\begin{array}{lll}
0 & \text { if } j \neq s \\
m(s)-1 & \text { if } & j=s
\end{array} .\right.
$$

Then

$$
\widehat{\mu}_{k}\left(\widetilde{\gamma}^{s}\right)=\alpha+\beta \exp (-1 / m(s))
$$

and hence

$$
\left|\hat{\mu}_{k}\left(\gamma^{s}\right)-\hat{\mu}_{k}\left(\widetilde{\gamma}^{s}\right)\right|=2 \beta \sin (2 \pi / m(s)) .
$$

If $3 \leqq m(s)<8 \pi$ and $\left|\hat{\mu}_{k}\left(\gamma^{s}\right)\right|<(1 / 100)$ then $\beta>(1 / 3)$ and

$$
\left|\hat{\mu}_{k}\left(\gamma^{s}\right)-\hat{\mu}_{k}\left(\widetilde{\gamma}^{s}\right)\right|>1 / 50
$$

and hence $\left|\hat{\mu}_{k}\left(\tilde{\gamma}^{s}\right)\right|>1 / 50$. Therefore we may conclude that for all $k$ either $\left|\hat{\mu}_{k}\left(\gamma^{s}\right)\right|$ or $\left|\hat{\mu}_{k}\left(\widetilde{\gamma}^{s}\right)\right|$ is greater than $1 / 100$ provided $m(s) \geqq 3$.

On $\Gamma^{k}, \hat{\mu}_{k}$ and $\hat{S}$ are identical. Suppose there is a $t$ so that 
for $\gamma \notin \Gamma^{t}$. Pick a $k>t$ so that there is an $s$ with $k>s>t$ for which $m(s) \geqq 3$. Then either $\left|\hat{\mu}_{k}\left(\widetilde{\gamma}^{s}\right)\right|$ or $\left|\hat{\mu}_{k}\left(\widetilde{\gamma}^{s}\right)\right|$ is greater than $1 / 100$. Hence $\left|\hat{S}\left(\gamma^{s}\right)\right|$ or $\left|\hat{S}\left(\tilde{\gamma}^{s}\right)\right|$ is greater than $1 / 100$ contradicting (1.19).

2. In this section we shall exhibit sets $E_{m}, F_{r}$ that do not have $A\left(E_{m}\right)$ or $A\left(F_{r}\right)$ isomorphic to $A\left(F_{r_{0}}\right)$ by standard isomorphisms. They are then not in the class $M_{y}$.

The first theorem is a converse to Lemma A.

TheOREM 2.1. If $\Sigma(1 / m(j))^{2}=\infty$, then $E_{m}$ is not an element of the class $M_{y}$.

Proof. It is sufficient to show that

$$
\sup _{\mu \in N(E)}\|\mu\|_{M \Lambda X} /\|\mu\|_{P M}=\infty
$$

since for $\nu \in N_{1}\left(F_{r_{0}}\right)\|\nu\|_{P M}=\|\nu\|_{\mathrm{MAX}}$. For each integer $s$, let $x^{s} \in \Pi Z_{m(j)}$ be that element with $x_{j}^{s}=\delta_{j}^{s}$. Let $\alpha_{s}$ be the two point measure

$$
\alpha_{s}\left\{x^{s}\right\}=\exp (1 / 3 m(s)) \text {. }
$$

For each $k$, define an element $\mu_{k}$ of $M\left(E^{k}\right)$ by

$$
\mu_{k}=\alpha_{1} * \cdots * \alpha_{k} \text {. }
$$

we see that

$$
\left\|\mu_{k}\right\|_{\mathrm{MAX}}=2^{k}
$$

while

$$
\left\|\mu_{k}\right\|_{P M}=\sup _{\xi_{s}} \mid \prod_{s=1}^{k}\left(1+\exp (1 /(3 m(s))) \cdot \xi_{s} \mid,\right.
$$

where the $\xi_{s}$ are $m(s)$ roots of unity. Since

$$
|1+\exp (1 / 3 m(s))| \geqq\left|1+\exp (1 / 3 m(s)) \xi_{s}\right|
$$

for $\xi_{s}$ any $m(s)$ root of unity, and since $\cos (\theta)<1-\theta^{2} / 4$ for $\theta<1$

$$
\begin{aligned}
\left\|\mu_{k}\right\|_{P M} & =2^{k} \prod_{s=1}^{k} \cos (\pi / 3 m(s)) \\
& \leqq 2^{k} \prod_{s=1}^{k}\left(1-(1 / 3 m(s))^{2}\right) .
\end{aligned}
$$

Therefore

$$
\left\|\mu_{k}\right\|_{\mathrm{XAX}} /\left\|\mu_{k}\right\|_{P M} \geqq 1 / \prod_{s=1}^{k}\left(1-(1 / 3 m(s))^{2}\right)
$$


and since $\Sigma(1 / m(s))^{2}=\infty,\left\|\mu_{k}\right\|_{\mathrm{MAX}} /\left\|\mu_{k}\right\|_{P M} \rightarrow \infty$ as $k \rightarrow \infty$.

We have actually shown more than claimed in Theorem 2.1. The proof shows that if $\{r(j)\}$ is any independent sequence and $\Sigma(1 / m(j))^{2}=$ $\infty$, then $A\left(E_{m}\right)$ is not isomorphic to $A\left(F_{r}\right)$ by a standard isomorphism.

The next theorem will imply that no condition on the convergence of $(r(j+1) / r(j))$ weaker than

$$
\Sigma(r(j+1) / r(j))^{2}<\infty,
$$

is sufficient for a set $F_{r}$ to be a member of the class $M_{y}$.

THEOREM 2.2. Suppose that $n_{j}$ is an increasing sequence of integers. Let $b \geqq 2$ be an integer and put $r(j)=b^{-n_{j}}$. If

$$
\Sigma(r(j+1) / r(j))^{2}=\infty
$$

then $F_{r}$ is not an element of the class $M_{y}$.

Proof. Let us assume for convenience that $\Sigma_{1}^{\infty}(r(2 j) / r(2 j-1))^{2}=\infty$ and $b=10$. We can also assume our set $F$ to be on the circle. For any integer $j$ define the two point measure $\gamma_{j}$ by

$$
\begin{gathered}
\gamma_{j}\{0\}=1 \\
\gamma_{j}\{r(j)\}=\exp \left(-\frac{1}{2}\right) .
\end{gathered}
$$

For each $k$, define an element $\nu_{k}$ of $M\left(F^{k}\right)$ by

$$
\nu_{k}=\gamma_{1} * \cdots * \gamma_{k} .
$$

Then for any integer $s$

$$
\left|\hat{\nu}_{2 k}(s)\right|=2^{2 k}\left|\prod_{1}^{2 k} \cos \left(\pi\left(s \cdot 10^{-n_{j}}-\frac{1}{2}\right)\right)\right| .
$$

In this product, consider terms $\delta_{j}(s)$ of the form

$$
\left|\cos \left(\pi\left(s \cdot 10^{-n_{2 j-1}}-\frac{1}{2}\right)\right) \cdot \cos \left(\pi\left(s \cdot 10^{-n_{2 j}}-\frac{1}{2}\right)\right)\right| .
$$

If

$$
\left|s \cdot 10^{-n_{2 j-1}}-\frac{1}{2}\right|<1 / 10 \bmod 1,
$$

then

$$
\left|s \cdot 10^{-n_{2 j}}-\frac{1}{2}\right| \geqq \frac{1}{10} \cdot\left(10^{n_{2 j-1}} / 10^{n_{2 j}}\right) \bmod 1 .
$$


Then

$$
\begin{aligned}
\left|\hat{\boldsymbol{\nu}}_{2 k}(s)\right| & =2^{2 k} \prod_{j=1}^{k}\left|\delta_{j}(s)\right| \\
& \leqq 2^{2 k} \prod_{j=1}^{k}\left(1-D \cdot\left(10^{n_{2 j}-1} / 10^{n_{2} j}\right)^{2}\right),
\end{aligned}
$$

where $D$ is an absolute constant. Therefore

$$
\left\|\nu_{2 k}\right\|_{P M} \leqq 2^{2 k} \prod_{j=1}^{k}\left(1-D(r(2 j) / r(2 j-1))^{2}\right) .
$$

However, $\left\|\nu_{2 k}\right\|_{\mathrm{MAX}}=2^{2 k}$, so

$$
\left\|\nu_{2 k}\right\|_{\text {MAX }} /\left\|\nu_{2 k}\right\|_{P M} \geqq\left|/ \prod_{j=1}^{k}\left(1-D(r(2 j) / r(2 j-1))^{2}\right)\right| .
$$

Therefore $\left\|\nu_{2 k}\right\|_{\text {MAX }} /\left\|\nu_{2 k}\right\|_{P M} \rightarrow \infty$ as $k \rightarrow \infty$. Hence $F_{r}$ is not a member of the class $M_{y}$. The proof with $b \neq 10$ is completely analogous to the proof with $b=10$.

The author wishes to thank Paul Cohen, Karel de Leeuw, Yitzhak Katznelson, and Carruth McGehee, for their counsel.

\section{BIBLIOGRAPHY}

1. N. Akhiezer, Theory of approximations, translated by C. J. Hyman, Ungar, New York, 1956.

2. A. Beurling and H. Helson, Fourier-Stieltjes transforms with bounded powers, Math. Scand. 1 (1953), 120-126.

3. H. Bohr, Almost periodic functions, translated by H. Cohn, Chelsea, New York, 1947.

4. J. W. S. Cassels, An introduction to diophantine approximation, Cambridge University Press, 1957.

5. K. DeLeeuw and Y. Katznelson, On certain homomorphisms of quotients of group algebras, Israel J. Math. 2 (1964), 120-126.

6. N. Dunford and J. T. Schwartz, Linear operators, Part I: General Theory, Interscience Publishers, New York, 1958.

7. J. P. Kahane and R. Salem, Ensembles parfaits et series trigonométriques, Hermann, Paris, 1963.

8. Y. Katznelson and W. Rudin, The Stone-Weierstrass property in Banach algebras, Pacific J. Math. 11 (1961), 253-265.

9. L. Loomis, An Introduction to abstract harmonic analysis, D. Van Nostrand, Princeton, 1953.

10. O. C. McGehee, Sets of uniqueness and sets of multiplicity, Israel J. Math. 2 (1966), 83-99.

11. Certain isomorphisms between quotients of a group algebra, Pacific J. Math. 21 (1967), 133-152.

12. Y. Meyer, Isomorphisms entre certaines algebres de restrictions, (to appear in Annales de L'Institut Fourier)

13. M. Naimark, Normed rings, translated by L. Boron, P. Noordhoff N. V., Sroningen, 1959. 
14. L. Pontrjagin, Topological groups, translated by E. Lehmer, Princeton University Press, Princeton, 1958.

15. W. Rudin, Fourier analysis on groups, Interscience Publishers, New York, 1962. 16. A. Zygmund, Trigonometric series, I and II, Cambridge University Press, London, 1959.

Received April 22, 1968, This work is part of a thesis for Stanford University supported by the U.S. National Science Foundation Graduate Fellowship Program.

CORNELL UNIVERSITY 



\section{PACIFIC JOURNAL OF MATHEMATICS}

\section{EDITORS}

H. ROYDEN

Stanford University

Stanford, California

Richard Pierce

University of Washington

Seattle, Washington 98105
J. DUGUNDJI

Department of Mathematics

University of Southern California

Los Angeles, California 90007

BASIL GORDON

University of California

Los Angeles, California 90024

\section{ASSOCIATE EDITORS}
E. F. BECKENBACH
B. H. NEUMANN
F. WOLF
K. YosHIDA

\section{SUPPORTING INSTITUTIONS}

UNIVERSITY OF BRITISH COLUMBIA

CALIFORNIA INSTITUTE OF TECHNOLOGY

UNIVERSITY OF CALIFORNIA

MONTANA STATE UNIVERSITY

UNIVERSITY OF NEVADA

NEW MEXICO STATE UNIVERSITY

OREGON STATE UNIVERSITY

UNIVERSITY OF OREGON

OSAKA UNIVERSITY

UNIVERSITY OF SOUTHERN CALIFORNIA
STANFORD UNIVERSITY

UNIVERSITY OF TOKYO

UNIVERSITY OF UTAH

WASHINGTON STATE UNIVERSITY

UNIVERSITY OF WASHINGTON

*

AMERICAN MATHEMATICAL SOCIETY CHEVRON RESEARCH CORPORATION TRW SYSTEMS

NAVAL WEAPONS CENTER 


\section{Pacific Journal of Mathematics}

\section{Vol. 31, No. $1 \quad$ November, 1969}

James Burton Ax, Injective endomorphisms of varieties and schemes........

Richard Hindman Bouldin, A generalization of the Weinstein-Aronszajn

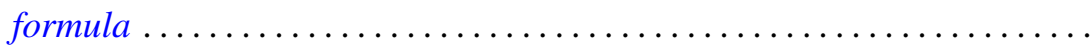

John Martin Chadam, The asymptotic behavior of the Klein-Gordon equation with external potential. II ...............................

Rina Hadass, On the zeros of the solutions of the differential equation

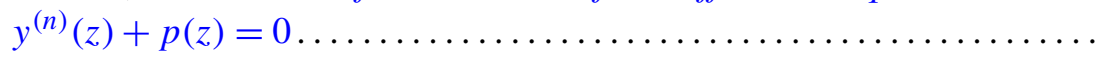

John Sollion Hsia, Integral equivalence of vectors over local modular lattices. II .............................................

Robert Hughes, Boundary behavior of random valued heat polynomial

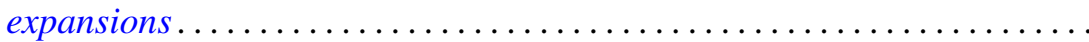

Surender Kumar Jain, Saad H. Mohamed and Surjeet Singh, Rings in which every right ideal is quasi-injective .........................

T. Kawata, On the inversion formula for the characteristic function .........

Erwin Kleinfeld, On right alternative rings without proper right ideals......

Robert Leroy Kruse and David Thomas Price, On the subring structure of

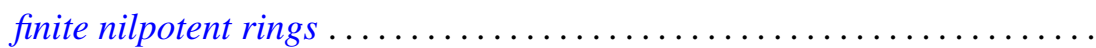

Marvin David Marcus and Stephen J. Pierce, Symmetric positive definite multilinear functionals with a given automorphism. .

William Schumacher Massey, Pontryagin squares in the Thom space of a bundle...

William Schumacher Massey, Proof of a conjecture of Whitney ...

John William Neuberger, Existence of a spectrum for nonlinear transformations

Stephen E. Newman, Measure algebras on idempotent semigroups ...

$\mathrm{K}$. Chandrasekhara Rao, Matrix transformations of some sequence spaces

Robert Bruce Schneider, Some theorems in Fourier analysis on symmetric

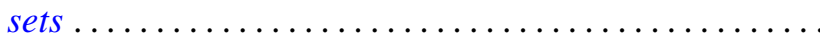

Ulrich F. K. Schoenwaelder, Centralizers of abelian, normal subgroups of hypercyclic groups...

Jerrold Norman Siegel, $G$-spaces, $H$-spaces and $W$-spaces

Robert Irving Soare, Cohesive sets and recursively enumerable Dedekind cuts...

Kwok-Wai Tam, Isometries of certain function spaces .... . .

Awadhesh Kumar Tiwary, Injective hulls of semi-simple modules over regular rings ....

Eldon Jon Vought, Concerning continua not separated by any nonaposyndetic subcontinuum .................... 